\title{
Radiation-Pressure Ejection of Planetary Nebulae in Asymptotic-Giant-Branch Stars
}

\author{
A.V. Sweigart \\ NASA Goddard Space Flight Center, Code 681, Greenbelt, MD 20771, \\ $U S A$
}

\begin{abstract}
We have investigated the possibility that radiation pressure might trigger planetary nebula (PN) ejection during helium-shell flashes in asymptotic-giant-branch (AGB) stars. We find that the outward flux at the base of the hydrogen envelope during a flash will reach the Eddington limit when the envelope mass $M_{\text {env }}$ falls below a critical value that depends on the core mass $M_{\mathrm{H}}$ and composition. These results may help to explain the helium-burning PN nuclei found in the Magellanic Clouds.
\end{abstract}

\section{Description of Radiation-Pressure Instability}

We have computed extensive AGB evolutionary sequences for heavy-element abundances $\mathrm{Z}=0.01716$ (solar) and 0.002 in order to study a radiation-pressure instability for ejecting a PN during a helium-shell flash. The existence of such an instability was previously confirmed by Wood \& Faulkner (1986) but only for more massive AGB stars with large core masses $\left(M_{\mathrm{H}}>0.86 M_{\odot}\right)$. In contrast, we find this instability at core masses as small as $M_{\mathrm{H}} \approx 0.6 M_{\odot}$ due, in part, to our use of the new OPAL opacities (Rogers \& Iglesias 1992).

Results for a typical sequence are presented in the left panels of Figure 1. The strong flashes in this sequence cause the hydrogen shell to expand outward to very low temperatures and densities. This, in turn, lowers the gas pressure and hence the minimum value $\beta_{\min }$ of the ratio $\beta$ of gas to total pressure near the base of the hydrogen envelope. Most importantly, we note from Figure 1 that $\beta_{\min }$ decreases monotonically from flash to flash and eventually goes to 0 , implying that the entire envelope is then supported by radiation pressure.

During the final flashes in Figure 1 the temperature within the hydrogen shell falls to $\log T=5.3$ at which there is a well-known peak in the OPAL opacities. When the shell reaches this peak, its opacity suddenly increases, and, as a result, its Eddington luminosity abruptly drops. This occurs at a time when the outward flux within the shell is considerably enhanced by the flash. As the shell continues to cool, its opacity increases further, thereby forcing an even greater expansion and driving $\beta$ down to even smaller values. The star thus encounters an "opacity catastrophe" whose likely outcome is envelope ejection.

The present sequences show that there is a critical $M_{\text {env }}$ at which radiation pressure will support the hydrogen envelope during a flash (see right panel of Figure 1). This critical $M_{\text {env }}$ increases linearly with $M_{\mathrm{H}}$ and decreases with decreasing metallicity, since the peak in the OPAL opacities is then smaller. 


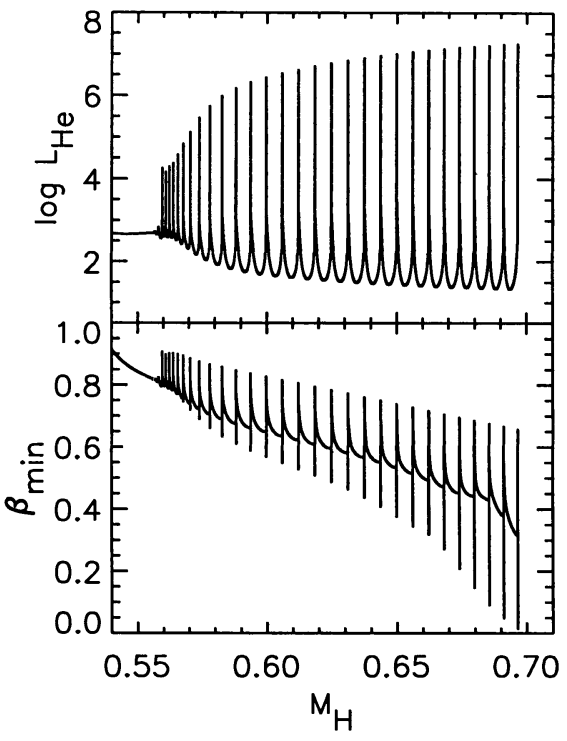

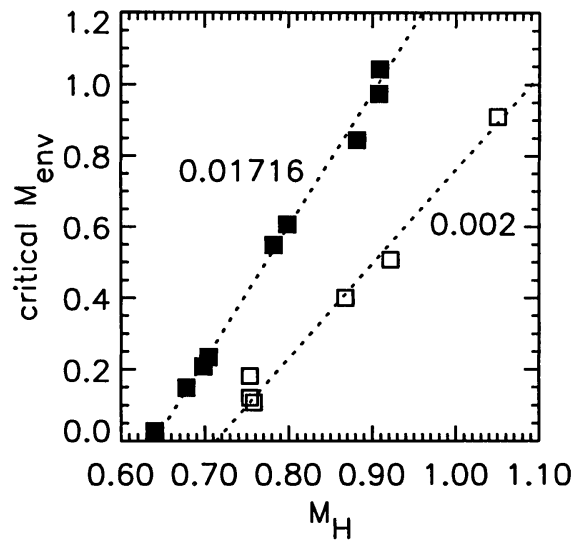

Figure 1. Helium-burning luminosity $L_{\mathrm{He}}$ in solar units (upper left panel) and minimum value $\beta_{\min }$ of the ratio $\beta$ of gas to total pressure near the base of the hydrogen envelope (lower left panel) as a function of the core mass $M_{\mathrm{H}}$ during the helium-shell flashes of a solar metallicity AGB star. The right panel shows the dependence of the critical envelope mass $M_{\mathrm{env}}$ at which $\beta$ goes to 0 during a flash on $M_{\mathrm{H}}$ for two heavy-element abundances: $\mathrm{Z}=0.01716$ (solid squares) and 0.002 (open squares). The dashed lines are linear fits to the model data.

The critical values of $M_{\text {env }}$ in Figure 1 represent the minimum envelope mass at which an AGB star can undergo a flash without $\beta$ going to 0 . These results depend, however, on the extent of 3rd dredge-up in the model calculations.

The minimum in $\beta$ during a flash occurs at the time when 3rd dredge-up is most likely. We suggest that such dredge-up might trigger PN ejection by increasing the opacity and thereby lowering the Eddington luminosity at the base of the envelope, especially at low metallicites where dredge-up is favored by current theoretical models. This might explain why the majority of halo PN's and most non-Type I PN's in the Magellanic Clouds are carbon rich. It might also explain why the PN K648 in M15 has a high carbon abundance despite the lack of AGB carbon stars in this globular cluster. We suggest therefore that the high carbon abundance of K648 was the cause of its PN ejection.

\section{References}

Rogers, F. J., \& Iglesias, C. A. 1992, ApJS, 79, 507

Wood, P. R., \& Faulkner, D. J. 1986, ApJ, 307, 659 